\title{
Double-channel fluorimeter with pulsed excitation of advanced glycation end products in skin
}

\author{
Vladimir N. Grishanov*, Victor S. Kulikov, and Konstantin V. Cherepanov \\ Samara National Research University, 34 Moskovskoye Shosse, Samara 443086, Russia \\ * e-mail: vladgrishanov@yandex.ru
}

\begin{abstract}
A double-channel diagnostic fluorimeter operating in pulsed mode for in vivo assessment of the content of advanced glycation end products (AGEs) in skin by the autofluorescence integral intensity in the visible spectral region is developed. To excite the fluorescence we use a light-emitting diode with the peak wavelength $365 \mathrm{~nm}$. A green light-emitting diode and an additional photodetection channel are intended to allow for the skin phototype of the patient. The fluorimeter analogue electronics comprises two photodetection channels based on silicon photodiodes. The accepted noise-suppressing circuitry solutions reduce the effect of sun and illumination lamp background light on the digital signal at the output of the 10-bit ADC to the one-bit level in both channels. The digital part of the fluorimeter is based on the Arduino platform. The software controls the operation modes of the fluorimeter, provides the quantitative processing of results, the diagnostic data storage and visualization. The experimental studies demonstrated the capabilities of revealing age-related changes in the skin. (C) 2018 Journal of Biomedical Photonics \& Engineering.
\end{abstract}

Keywords: skin autofluorescence; fluorimeter; advanced glycation end products; medical diagnostics.

Paper \#3274 received 15 Jan 2018; revised manuscript received 7 Mar 2018; accepted for publication 12 Mar 2018; published online 31 Mar 2018. doi: 10.18287/JBPE18.04.010506. [Special Section. Workshop "Biophotonics" of the XV all-Russian Youth Samara conference-contest on optics and laser physics].

\section{References}

1. R. V. Golubev, G. V. Papayan, A. A. Glazunova, N. Yu. Korosteleva, N. N. Petrishchev, and A. V. Smirnov, "Study of skin autofluorescence for determining the content of glycation end products in patients under chronic haemodialysis," Terapevticheskii arkhiv 88(6), 65-72 (2016) [in Russian].

2. D. A. Rogatkin, O. A. Prisnyakova, L. G. Moiseeva, and A. S. Cherkasov, "Analysis of the accuracy of clinical laser fluorescence diagnostics," Measurement Technique 41(7), 670-674 (1998).

3. I. A. Novikov, Ya. O. Grusha, and N. P. Kiryushchenkova, "Increasing the efficiency of fluorescence diagnostics of skin and mucosa neoplasms in ophthalmic oncology," Vestnik RAMN 10, 62-69 (2012) [in Russian].

4. A. V. Dunaev, V. V. Dryomin, E. A. Zherebtsov, S. G. Palmer, S. G. Sokolovskiy, and E. U. Rafailov, "Analysis of individual variability of parameters in laser fluorescence diagnostics," Biotekhnosfera 2(26), 3947 (2013) [in Russian].

5. R. B. Blyumin, E. M. Naumova, and A. A. Khadartsev, "Technologies of non-contact diagnostics," Vestnik novykh meditsinskikh tekhnologiy XV(4), 146-149 (2008) [in Russian].

6. R. Meerwaldt, R. Graaff, P. H. N. Oomen, T. P. Links, J. J. Jager, N. L. Alderson, S. R. Thorpe, J. W. Baynes, R. O. B. Gans, and A. J. Smit, "Simple non-invasive assessment of advanced glycation end product accumulation," Diabetologia 47(7), 1324-1330 (2004).

7. D. J. Mulder, P. L. van Haelst, R. Graaff, R. O. Gans, F. Zijlstra, and A. J. Smit, "Skin autofluorescence is elevated in acute myocardial infarction and is associated with the one-year incidence of major adverse cardiac events," Netherlands Heart Journal 17(4), 162-168 (2009). 
8. R. Meerwaldt, J. W. L. Hartog, R. Graaff, R. J. Huisman, T. P. Links, N. C. den Hollander, S. R. Thorpe, J. W. Baynes, G. Navis, R. O. B. Gans, and A. J. Smit, "Skin autofluorescence, a measure of cumulative metabolic stress and advanced glycation end products, predicts mortality in hemodialysis patients," Journal of the American Society of Nephrology 16(12), 3687-3693 (2005).

9. G. V. Papayan, N. N. Petrishchev, E. V. Krylova, K. Uk, S. V. Kim, V. B. Berezin, and B. Soo-Jin, "Method of estimating the biological age of skin by means of a fluorescence multispectral video dermatoscope," Journal of Optical Technology 77(2), 126-131 (2010).

10. M. S. Ahmad, T. Kimhofer, S. Ahmad, M. N. AlAma, H. H. Mosli, S. I. Hindawi, D. O. Mook-Kanamori, K. Šebeková, Z. A. Damanhouri, and E. Holmes, "Ethnicity and skin autofluorescence-based risk-engines for cardiovascular disease and diabetes mellitus," PLoS One 12(9), e0185175 (2017).

11. K. Uk, V. B. Berezin, G. V. Papayan, N. N. Petrishchev, and M. M. Galagudza, "Spectrometer for fluorescence-reflection biomedical research," Journal of Optical Technology 80(1), 40-48 (2013).

12. N. N. Bulgakova, V. V. Smirnov, V. I. Fabelinskii, A. G. Fedotov, and S. V. Shchichkin, "Spectral fluorescence colposcope," Biomeditsinskaya radioelektronika 4, 42-49 (2013) [in Russian].

13. D. V. Kornilin, and V. N. Grishanov, "Portable fluorescence meter for medical applications," Proceedings of SPIE 9887, 98871N (2016).

14. D. V. Kornilin, V. N. Grishanov, V. P. Zakharov, and D. S. Burkov, "Portable fluorescence meter with reference backscattering channel," Proceedings of SPIE 9961, 99610C (2016).

15. O. I. Kozlov, E. A. Zherebtsov, A. I. Zherebtsova, V. V. Dryomin, and A. V. Dunaev, "Laser Doppler flowmetry method and device for recording the intensity of the skin blood flow components," Biomeditsinskaya radioelektronika 6, 68-75 (2017) [in Russian].

16. I. A. Nakhaeva, O. A. Zyuryukina, M. R. Mohammed, and Yu. P. Sinichkin, "The effect of external mechanical compression on in vivo water content in human skin," Optics and Spectroscopy 118(5), 834-840 (2015).

17. I. A. Nakhaeva, M. R. Mohammed, O. A. Zyuryukina, and Yu. P. Sinichkin, "The effect of an external mechanical compression on in vivo optical properties of human skin," Optics and Spectroscopy 117(3), 506512 (2014).

18. Y. Wang, L. Zhang, L. Zhu, Y. Liu, G. Zhang, and A. Wang, "A trifurcated fiber-optic probe based optical system designed for AGEs measurement," Proceedings of SPIE 8329, 832908 (2012).

19. G. T. Petrovsky (Ed.), Coloured Optical Glass and Special Glasses. A Catalogue, Dom optiki, Moscow (1990) [in Russian].

20. V. A. Petin, Project Using Arduino Controller, BKhV Peterburg, Saint-Petersburg (2014) [in Russian].

21. G. Petin, "Key synchronous detector," Skhemotekhnika 3, 14-15 (2003) [in Russian].

\section{Introduction}

The intensity of induced endogenous skin fluorescence, or autofluorescence (AF), is measured in vivo for diagnostic purposes in endocrinology, cardiology, and nephrology [1-8]. Complications of diabetes mellitus, coronary heart disease, and renal insufficiency lead to the increased concentration of advanced glycation end products (AGEs) in the skin, manifesting itself in the excess intensity of the skin AF. The processes of aging [9] and biological oxidation [5] of tissues are also accompanied by the change of AGE content in the skin.

The advanced glycation end products are a result of the reaction of sugars with proteins without enzyme participation. In collagen that contains a large amount of glucose, the AGE manifests itself in the increased number of covalent-bond cross-links [1]. The structures most probably responsible for the fluorescence intensity growth due to AGE include CDCCL (collagenasedigestible cross-links) and ECL (elastin cross-links), in which the long-wave edges of the excitation and emission bands amount to $360 / 420 \mathrm{~nm}$ and $390 / 420 \mathrm{~nm}$, respectively. With age, the rate of ECL accumulation can slightly exceed that of CDCCL. Another cause of such dynamics can be the accumulation of lipofuscin pigment. Its fluorescence maximum lies in the green spectral region and the lipofuscin itself is known as an age pigment. It is worth noting that the most probable AGEs responsible for the increased level of autofluorescence, such as CDCCL, ECL, and pentosidine form a certain group of substances with similar properties, including the fluorescent ones [6, 9].

To assess the AGE content they use either different modifications of AGE-Reader devices [10], based on measuring the AF spectrum, or original all-purpose fluorimeters having a complex transformable structure $[4,5,11,12]$. Since the originality and universality cannot provide operational and economic efficiency, the development of portable and easy-to-operate instrumentation for measuring the integral intensity of skin fluorescence is rather urgent.

The portable fluorimeter is based on measuring the integral parameter of the AGE content in skin, proposed in Refs. $[6,7]$. The idea is to calculate the ratio of the measured value of the AF intensity in the wavelength range $420-600 \mathrm{~nm}$ to the intensity of the fluorescenceexciting radiation in the wavelength range $300-420 \mathrm{~nm}$, elastically scattered by skin. The use of an integral criterion allowed essential simplification of the 
diagnostic fluorimeter engineering solution by means of using two photodiodes in the receiving part, one of them installed after a cut-off optical absorption filter. This photodiode provides the analogue integration of the signal over the AF spectrum. The other photodiode records the signal of the AF-exciting ultraviolet (UV) radiation scattered elastically by the skin. At the expense of high sensitivity of photodetection channels, it was enough to excite AF with a low-power UV photodiode with purifying optical filter. This allowed the creation of a compact optical unit with low energy consumption and low supply voltage.

Earlier the authors proposed capable versions of diagnostic fluorimeters with continuous excitation [13] and additional channel for elastic scattering [14]. However, in the process of trial exploitation the effect of bright sunlight illumination on the instrument indications was revealed, although the forearm of the examined patient shielded the entrance window of the device. This effect is probably due to the background radiation arriving at the high-sensitivity detection channel after multiple scattering in the skin. Besides that, we observed intrinsic fluorescence from the cut-off optical filter made of coloured glass FGL435 (Thorlabs, USA), overlapping the AF spectrum of the skin.

In the integral assessment of AGE content in skin, one should allow for the significant individual variability of $\mathrm{AF}$ and elastic scattering signals [4], which can reduce the reliability of diagnostics. The probable causes of the individual variability include the endogenous melanin [11], the saturation of skin with blood microvessels at the measurement site [4], the vessels blood filling [15], and the skin tissue mechanical compression $[16,17]$. Therefore, it looks promising to introduce additional information channels that would allow for the individual features of radiation absorption in skin. In particular, the authors of Ref. [18] proposed additional measurement of spectral intensity of radiation from a white light-emitting diode (LED) scattered by skin. However, the interpretation of the results for such wideband signal gives rise to certain difficulties in the measurement of the integral diagnostic parameter. In this case, it is reasonable to perform the measurements in the centre of the fluorescence band, using an additional coloured LED for this purpose.

The purpose of the reported study was to develop a version of the fluorimeter in which the individual variability of the integral diagnostic parameter, the effect of external illumination, and the fluorescence of its own optical elements would be reduced. This reduction was achieved by measuring the skin-scattered radiation in the visible spectral region using pulsed sources of radiation, frequency filtering in the photodetection channels, and low-AF materials in the construction of the fluorimeter optical scheme.

\section{Functional diagram of the fluorimeter}

The functional diagram of the fluorimeter with pulsed excitation is presented in Fig. 1. The excitation of AF of the AGE is implemented using the radiation of the EOLD-365-525 UV LED 1 having the peak wavelength near $365 \mathrm{~nm}$ that passes through the purifying optical filter 2 made of coloured $3 \mathrm{~mm}$ thick optical UFS6 glass. Detailed description of the function and choice justification of the coloured optical glass UFS6 is given in Ref. [14]. The UV radiation purified from the longwave component 3 through the window in the metallic case of the device 4 , covered with the protective glass 5 (microscope slide $1 \mathrm{~mm}$ thick), is incident on the fluorescing object (forearm inner side) 6, applied to the glass plate from outside.

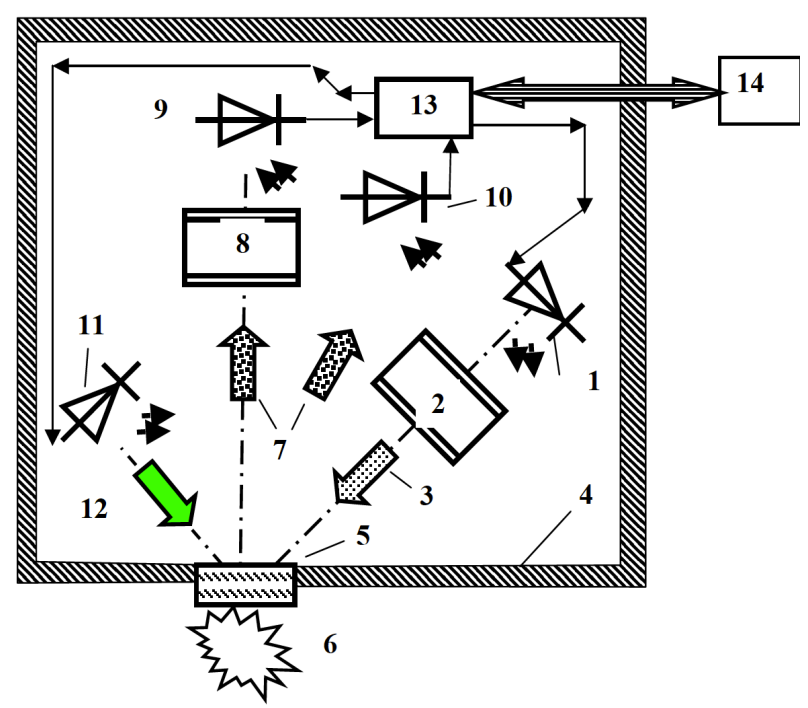

Fig. 1 Functional diagram of the alternating-current fluorimeter: 1 - light-emitting diode EOLD-365-525; 2 - purifying optical filter made of coloured optical glass UFS6; 3 - ultraviolet radiation flux; 4 - metallic case with a window; 5 - protection glass; 6 - object of study; 7 - flux of radiation scattered towards the photodetectors; 8 - cut-off optical ZhZS6 filter; 9 - BPW21R photodiode; 10 - SFH 229 photodiode; 11 - DFL-3014PGW green LED; 12 - flux of green radiation; 13 - electronics unit; $14-\mathrm{PC}$; thin arrows show the electric connections and the directions of electric signal transmission.

A part of the exciting radiation scattered by the forearm skin and the fluorescence radiation 7 passes through the cut-off optical filter 8 made of coloured $3 \mathrm{~mm}$ thick ZhZS6 glass, in which the elastically scattered UV radiation is almost completely absorbed. The ZhZS6 glass does not belong to fluorescent materials [19] and, therefore, does not contribute to the recorded signal, which was confirmed experimentally. Thus, only the fluorescence radiation is incident on the silicon BPW21R photodiode 9, so that the output electric signal from this photodiode is directly proportional to the integral intensity of the skin AF in the transmission band of the filter 3. A part of elastically 
scattered and fluorescence radiation is incident on the SFH229 photodiode 10 . Since the quantum efficiency of AGE fluorescence is small, we assume that the output signal from the SFH229 photodiode is proportional to the intensity of the exciting UV radiation, scattered by the skin. This photodiode belongs to the channel of the elastic scattering intensity measurement.

To reduce the variability of the integral diagnostic parameter, the green light-emitting diode DFL-3014PGW 11 with the peak wavelength $539 \mathrm{~nm}$ was introduced into the optical scheme of the fluorimeter.

The UV and the green LED are software switched on by turns and operate in pulsed mode with the pulse repetition rate $487 \mathrm{~Hz}$, which allowed the synthesis of the receiving electronics for the alternating-current operation. The axes of the directional patterns of the UV and green LED make an angle $\sim 45^{\circ}$ with the normal to the protection glass 5 . Thus, the $45^{\circ} / 0^{\circ}$ geometry of measuring the fluorescence and the elastic scattering is accepted. The functional diagram is completed with the unit of electronics 13 and the personal computer 14 .

\section{The fluorimeter electronics unit}

The fluorimeter electronics unit comprises two photodetector channels, two drivers of photodiodes, and the Arduino board [20]. The first photodetection channel is optimised for measuring the fluorescence intensity and the second channel for measuring the intensity of elastically scattered exciting UV radiation and the radiation of the greed LED. The LED drivers provide them with stable-amplitude currents, using the source of reference voltage. The Arduino board determines by software the sequence of switching the LEDs on, the repetition rate, and the off-duty ratio of the optical pulses.

A specific feature of the channel recording the fluorescence radiation is the system of active noise suppression, in which the frequency filtering method is implemented. As frequency-selective element the lockin-amplifier [21] is used, since the fluorimeter comprises a source of reference pulsed signal.

In the skin AF fluorescence intensity measuring channel, the BPW21R photodiode operating in the photoconductive mode is connected to the transimpedance amplifier (current-voltage convertor), the signal from which is preliminarily amplified by the bandpass amplifier and arrives at the synchronous detector. The role of electronic analogue key is played by the CMOS multiplexor operating in the analogue mode. The electronic key is controlled by the same clock signal from the microcontroller unit, as the illuminator driver (that provides the synchronous mode of operation). The signal from the outputs of the electronic key arrives at the inputs of the instrumentation amplifier, where the detection occurs. The detected signal is applied to the input of the active low-pass filter. In the presented device, we use the active low-pass Sallen-Key filter of the second order, the nominals of the RC-components of which provide the Q-factor equal to 16. The electronic key, the instrumentation amplifier, and the low-pass filter comprise the synchronous detector. The detected filtered signal arrives at the final amplifier, from which the signal is transmitted to the analogue-to-digital convertor (ADC) of the Arduino board. The final amplifier executes the scaling function in order to use the entire dynamic range of the ADC.

In the channel measuring the intensity of the elastically scattered radiation, the silicon SFH229 photodiode is used as a photosensitive element. The level of the recorded signal in this case is relatively high, and no additional noise-suppression system is necessary. One should only match the maximal level of the output signal with the ADC dynamic range and suppress the background radiation from the natural and artificial illumination sources. The channel consists of the transimpedance amplifier of alternating voltage and the peak amplitude detector based on diodes with doubling (to enhance the linearity, the Schottky diodes with small forward voltage drop are used). The detected signal is applied to the input of the second ADC of the Arduino board.

\section{Fluorimeter construction}

Structurally the fluorimeter consists of a remote unit, an additional $12 \mathrm{~V}$ power supply, and a PC. The base for mounting the electronics 2 is the bottom cover 1 of the case (Fig. 2). In the top cover of the case, a $\varnothing 10 \mathrm{~mm}$ window is drilled that serves for exciting the patient's skin fluorescence and for receiving the fluorescence and elastic scattered radiation. The optical-to-electrical (OE) unit of the fluorimeter 4 is mounted coaxial with the window. The OE unit carrying the LEDS and the photodiodes determines the geometry of detecting the intensity of fluorescence and elastic scattering.

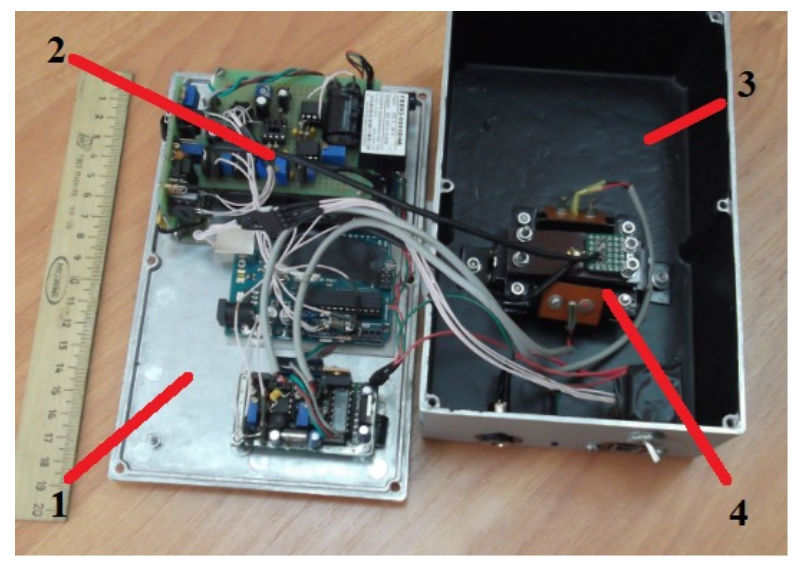

Fig. 2 Construction of the fluorimeter remote unit: 1 - bottom cover; 2 - boards of electronics unit; 3 - top cover of the case; 4 - optical-to-electrical unit.

For performing diagnostic procedures, the remote unit is placed on a table with its window up. The inner side of the patient's forearm is applied to the window, although, in principle, the remote unit can be applied 
itself to any part of a human body. The case of the remote unit has the dimensions $153 \times 83 \times 51 \mathrm{~mm}$.

The fluorimeter software creates and saves on a disc of the controlling PC the file, containing the information on the patient, the date and time of the diagnostic procedure, the values of signals (counts) from the ADC of the Arduino platform in arbitrary units for the UV or green LED switched on. The file also contains the results of statistical processing of the signals, in particular, the arithmetic mean amplitudes during the measurement time for the signals of autofluorescence, $M F$, elastic scattering with the UV LED switched on, $M E_{U V}$, elastic scattering with the green LED switched on, $M E_{G}$; the coefficients of variation for the signals of autofluorescence, $V F$, elastic scattering with the UV LED switched on, $V E_{U V}$, elastic scattering with the green LED switched on, $V E_{G}$; the mean amplitude ratios of autofluorescence to elastic scattering in the UV range, $M R_{U V}$, autofluorescence to elastic scattering in the green range, $M R_{G}$, and autofluorescence signal to the product of elastic scattering signals in the UV and green range, $M R_{U V G}$.

\section{Results of the fluorimeter testing}

The fluorimeter was calibrated using the black body model (BBM) and the fluoroplastic sample - a certainly non-fluorescing object with stable optical properties. The experiments with BBM were aimed to estimate the contribution of the electronic tract noises, the radiation scattered by the fluorimeter elements, and the cross-talk of channels due to imperfections of the purifying and cut-off optical filter. The following results were obtained. With the UV LED switched on at the output of the fluorescence channel we got $M F=68$ arb. units with the coefficient of variation $V F=3 \%$. At the output of the elastic scattering channel we obtained $M E_{U V}=14$ arb. units with the coefficient of variation $V E_{U V}=5 \%$. With the green LED switched on at the output of the elastic scattering channel we got $M E_{G}=168$ arb. units with the coefficient of variation $V E_{G}=0,4 \%$. Note that during the green LED operation the fluorescence channel was automatically switched off to prevent saturation. In the subsequent experiments, we interpreted the above values as steady-state noises with constant arithmetic mean values and programmatically subtracted them.

In the fluorimeter trial with the stable fluoroplastic scatterer and the UV LED switched on, no significant signal increase at the output of the fluorescence channel was observed, and the coefficient of variation amounted to $V F=4 \%$, which differs from the BBM experiments by $1 \%$, i.e., within the measurement error. The mean value of the signal at the output of the elastic scattering channel increased to $M E_{U V}=41.5$ arb. units, the coefficient of variation being $V E_{U V}=1.7 \%$. With the green LED switched on, the mean value increased to $M E_{G}=318.2$ arb. units, the coefficient of variation being $V E_{G}=0.21 \%$. The external background illumination, caused by the sunlight and the illuminating lamps did not manifest itself in the output signal.
Thus, the instability of the optical-to-electrical system of the fluorimeter that can be considered as the instrumentation error does not exceed 2 arb. units.

The individual variability of the results and the stability of the fluorimeter indications were estimated by repeating multiple measurements at nearly the same site of the left forearm inner side of the same volunteer (Table 1). The first series of measurements simulated the standard diagnostic procedure and was repeated during 2.5 months every three days at nearly the same time of the day. The second and the third series were performed during one hour with removing the forearm from the window before each new measurement. The fourth series of measurements was performed without removing the forearm from the window to demonstrate the stability of indications.

Table 1 Individual variability of the measurement results.

\begin{tabular}{ccccc}
\hline \multirow{2}{*}{$\begin{array}{c}\text { No. of } \\
\text { the } \\
\text { series }\end{array}$} & $\begin{array}{c}\text { The number } \\
\text { of } \\
\text { experiments } \\
\text { in the series }\end{array}$ & \multicolumn{3}{c}{$\begin{array}{c}\text { Coefficient of } \\
\text { variation }\end{array}$} \\
\cline { 3 - 5 } & $\begin{array}{c}V F, \\
\%\end{array}$ & $\begin{array}{c}V E_{U V}, \\
\%\end{array}$ & $\begin{array}{c}V E_{G}, \\
\%\end{array}$ \\
\hline 1 & 28 & 13 & 20 & 22 \\
\hline 2 & 8 & 10 & 8 & 4 \\
\hline 3 & 10 & 4 & 6 & 3 \\
\hline 4 & 8 & 1.0 & 2.1 & 1.7 \\
\hline
\end{tabular}

Comparing the values of the variation coefficients obtained in the series with removing the forearm from the window, one can see that a considerable contribution to the variability of indications arises from uncontrollable changes in the conditions of the experiment, caused by the change of the chosen area of exposure and the forearm position. The series 4, performed without removing the forearm, demonstrates the stability of the instrument indications.

In 58 practically healthy persons aged from 16 to 65 years, a comparative experiment on the stability of the diagnostic parameters $M R_{U V}$ and $M R_{U V G}$ with respect to the endogenous melanin was carried out. The authors proceeded from the fact that the skin of the outer side of forearm contains greater concentration of melanin as compared to the inner side. This fact can be easily confirmed visually, since the outer side of the forearm always looks more suntanned, and experimentally, by the smaller values of the signals $M F_{U V e}, M E_{U V e}, M E_{G e}$ for the outer side in comparison with their values $M F_{U V i}$, $M E_{U V i}, M E_{G i}$ for the inner side.

The experiment was carried out as follows. The inner and outer side of the forearm were alternately applied to the input window of the device. As a result, the values of the diagnostic parameters were measured for the inner $\left(M R_{U V i}\right.$ and $\left.M R_{U V G i}\right)$ and outer $\left(M R_{U V e}\right.$ and $\left.M R_{U V G e}\right)$ sides of the forearm of each arm, and the absolute values of the relative differences of these diagnostic parameters were calculated: 
Table 2 Age-related changes of the normalised ratios.

\begin{tabular}{c|c|c|c|c|c|c|c|c}
\hline $\begin{array}{c}\text { Number } \\
\text { of tested } \\
\text { persons }\end{array}$ & $\begin{array}{c}\text { Age } \\
\text { interval, } \\
\text { years }\end{array}$ & $\begin{array}{c}\text { Interval } \\
\text { centre, } \\
\text { years }\end{array}$ & $\begin{array}{c}R F_{U V}, \\
\text { arb. units }\end{array}$ & $\begin{array}{c}\text { Coefficient } \\
\text { of variation, } \\
\%\end{array}$ & $\begin{array}{c}R F_{G}, \\
\text { arb. } \\
\text { units }\end{array}$ & $\begin{array}{c}\text { Coefficient } \\
\text { of variation, } \\
\%\end{array}$ & $\begin{array}{c}R F_{U V G}, \\
\text { arb. units }\end{array}$ & $\begin{array}{c}\text { Coefficient } \\
\text { of } \\
\text { variation, } \\
\%\end{array}$ \\
\hline 47 & $16-25$ & 20 & 0.59 & 23 & 0.65 & 33 & 0.60 & 30 \\
\hline 9 & $26-35$ & 30 & 0.67 & 35 & 0.84 & 17 & 0.62 & 44 \\
\hline 4 & $36-45$ & 40 & 0.71 & 12 & 0.55 & 22 & 0.77 & 8 \\
\hline 4 & $46-55$ & 50 & 0.71 & 11 & 0.95 & 33 & 0.67 & 23 \\
\hline 7 & $56-65$ & 60 & 1.0 & 27 & 1.0 & 36 & 1.0 & 34 \\
\hline
\end{tabular}

$D R_{U V}=\left|2\left(M R_{U V i}-M R_{U V e}\right) /\left(M R_{U V i}+M R_{U V e}\right)\right|$,

$D R_{U V G}=\left|2\left(M R_{U V G i}-M R_{U V G e}\right) /\left(M R_{U V G i}+M R_{U V G e}\right)\right|$.

The arithmetic mean values of the relative differences for 58 patients amounted to $\mathrm{M}\left(D R_{U V}\right)=0.28$ and $\mathrm{M}\left(D R_{U V G}\right)=0.18$, i.e., $\mathrm{M}\left(D R_{U V}\right) / \mathrm{M}\left(D R_{U V G}\right)=1.5$, which allows a conclusion that the diagnostic parameter $M R_{U V G}$ is less sensitive to such destabilising factor as endogenous melanin.

Table 2 summarises the measurement data for the patients divided into age groups, with the standard statistical processing of the results performed within each group. The measured values of $M R_{U V}, M R_{G}$, $M R_{U V G}$ were preliminarily normalised to the maximal value in the group:

$$
\begin{aligned}
& R F_{U V}=M R_{U V} / M R_{U V, \text { max }}, \\
& R F_{G}=M R_{G} / M R_{G, \text { max }}, \\
& R F_{U V G}=M R_{U V G} / M R_{U V G, \max } .
\end{aligned}
$$

Then using the least squares method, the parameters for the linear regression equation were determined for each normalized mean value of the signals (3) - (5):

$$
y(t)=k_{1} t+k_{0}
$$

where $t$ is the age of a healthy patient expressed in years. The values of $k_{1}$ and $k_{0}$ for each of the functions $\left[R F_{U V}(t), R F_{G}(t), R F_{U V G}(t)\right] \equiv y(t)$ are also presented in Table 2.

The obtained trend of age-related changes of AGE agrees with the results of Refs. $[6,9,14]$ for all the three ratios $M R_{U V}, M R_{G}, M R_{U V G}$ due to the small difference of the linear regression parameters (see Table 2). One can see that in the case of considering only the scattering and absorption in the fluorescence emission band, the ratio $R F_{G}$ is characterised by relatively large coefficients of variation. However, for the simultaneous consideration of the skin absorption and scattering in the UV and visible spectral region, $R F_{U V G}$ weakly differs from the values obtained considering only the individual skin properties at the excitation wavelength, $R F_{U V}$. The latter is probably due to two reasons: the greater intensity of the exciting UV radiation and the limited statistical data, which does not allow unambiguous preference of one of the normalisation methods.

\section{Conclusion}

A fluorimeter intended for in vivo diagnostics of the content of fluorescent advanced glycation end products in human skin is designed, its characteristics are experimentally studied and the trial measurements are carried out. The use of pulsed radiation sources made it possible to improve the noise immunity of the fluorimeter against the background illumination, to normalise the integral diagnostic parameter allowing for the skin individual optical properties and phototype in the visible and UV spectral regions, to determine the individual variability of skin AF, and to reveal its agerelated changes. We demonstrate that the integral diagnostic parameter, defined as the ratio of the fluorescence intensity to the product of intensities of the elastic scattering in the ultraviolet and green spectral regions, is more robust against the variations endogenous melanin content in skin than two other ratios (the fluorescence to UV or green scattering separately).

\section{Disclosures}

The authors have no relevant financial interests in this article and no potential conflicts of interest to disclose.

\section{Acknowledgment}

The work was supported by the Russian Foundation for Basic Research, grant No. 17-42-630907r_a. 